\title{
Active Management of a Heterogeneous Energy Store for Electric Vehicles
}

\author{
Alexander Styler, Gregg Podnar, Paul Dille, Matthew Duescher, Christopher Bartley, Illah Nourbakhsh
}

\begin{abstract}
The successful introduction of electric vehicles continues to be stifled by the high cost and limited performance life of battery technology. We assert that a disruptive improvement in systems-level cost-of-performance is possible by employing a rate-heterogeneous energy storage system, combining low-rate batteries and high-rate supercapacitors, that is mated to a predictive control system that optimizes power management by exploiting topographic information, traffic history, and specific driver performance. Such predictive power management, optimizing energy storage throughout episodes of vehicle acceleration and regenerative braking, has the potential to significantly decrease the total energy duty on the vehicle's batteries.
\end{abstract}

\section{INTRODUCTION}

There is a new climate of viability for the electric vehicle. However, significant barriers to entry, including cost-ofownership and driving range, still limit the potential growth of this sector. These barriers stem from deficiencies in battery technology: in traditional systems, the high-current, highcycle demands of electric vehicles require new expensive chemistries such as thin electrode Lithium-ion, and battery degradation due to heavy duties shorten both the lifetime and range of vehicles. The ChargeCar project seeks to remove both barriers to entry using a heterogeneous energy storage system coupled with intelligent energy management algorithms. The system introduces a small supercapacitor in order to act as a high-rate, intelligent buffer fronting a lowerrate, high capacity battery. Charge can be moved between the stores and the electric motor bidirectionally, creating a degree of freedom that can be exploited to maximize efficiency in the system. Algorithms to control the energy flow in the system can utilize information such as vehicle state, driver history, GPS coordinates, and eventually even internet-available services such as traffic data, construction warnings, and weather reports.

\section{A. Heterogeneous Energy Storage System for Electric Vehi-} cles

The opportunity to make significant improvements in electric vehicle energy storage and power management systems is expanded when combining both high energy density and high power density components into a managed energy storage system [1]. Battery technology improvements along both of these vectors is ongoing but there are electrochemical tradeoffs between power rate and energy availability as well as

This work was supported by Donna Auguste and David Hayes, Bombardier Inc., Google, and The Heinz Endowments

Authors are with the Robotics Institute, Carnegie Mellon University, Pittsburgh, PA 15213, USA astylereri.cmu.edu significant battery life impacts. Furthermore, homogeneous solutions that select a single battery chemistry that has both sufficient energy density and sufficient power density for electric vehicle commuting bias the designer toward solutions that have high cost and operate at the extremal points of the existing battery performance space.

The alternative is to consider a heterogeneous system in which high energy and high power density are achieved with separate technologies. This approach has been previously explored with promising results for various hybrid electric vehicles, including both hydrogen and petrol fueled vehicles [2]. These hybrids usually use the electric subsystem to handle the high-rate demands, allowing the other subsystem to operate in a maximally efficient manner [3].

We consider the combination of high power density, high cycle life supercapacitors and high energy density batteries, as shown in Fig. 1. When the maximum rate of power draw (or recharge) of a secondary battery is constrained, the total energy that can be recovered from a charge, as well as the usable cycle life of the battery are both increased. Moderaterate batteries are far less expensive as they need not rely on exotic chemistries, plate materials, or manufacturing processes. While supercapacitors offer relatively low energy densities, they have extremely high power densities as well as delivering life-of-the-vehicle service (nominally one million full charge/discharge cycles). Therefore, in an electric vehicle where both high power density is required for acceleration and high energy density is required for practical range, a combination of batteries and supercapacitors can meet the demand. A heterogeneous energy store could alternatively consist of two different battery chemistries, and the theoretical nature of this work is agnostic to properties of the highrate component. The integration of supercapacitors into an electric vehicle has been previously shown to have promising results on the Federal Urban Driving Schedule (FUDS) [4], but relied on an unrealistically large supercapacitor array, and was incredibly specific in its energy management to only handle the FUDS cycle.

Simply using the high-power supercapacitor as a buffer, with energy only being stored from regenerative braking then spent greedily, will also have very limited success due to the loss of energy from friction and inefficiencies in the system. Ultimately, the energy gathered from regenerative braking is not sufficient, and the battery will ultimately have to be tapped directly for high-power acceleration cycles whenever the supercapacitor is empty. Instead of this or a specific, constant, energy transfer rate that will only handle the FUDS cycle in a maximally efficient manner, an intelligent 
algorithm can be used to actively predict upcoming duty for any trip and transfer energy accordingly.

\section{B. Potential Gains from Proposed Storage System}

A combination energy storage system composed of moderate-rate secondary batteries and supercapacitors leverages the best qualities of each energy storage technology. By shifting the high-current duty for acceleration and regenerative braking from the battery to a supercapacitor, a number of factors are significantly changed. Battery degradation due to high-current discharge and charge, total charge served, and heating is substantially reduced [5]. Additionally, highcurrent resistive losses of energy in the battery are reduced, yielding a direct increase in vehicle range. Other gains in range may be observed as the battery pack will stay cooler due to reduced high-power cycles, allowing the pack to operate in its most efficient range and saving energy otherwise spent on active cooling. These changes increase battery pack longevity as well as allow the use of battery chemistries that are less expensive, lowering total-cost-of ownership across the board. This approach also allows more of the battery available energy to be utilized, potentially allowing a smaller battery pack for the same range. A smaller battery pack in turn translates into a lighter vehicle, also increasing range and decreasing the logistical tail.

\section{Intelligent Power Demand Prediction to Maximize System Efficiency}

Optimal management of this compound energy system requires intelligent algorithms to predict energy demands and adjust the energy stored in the supercapacitor to maximize efficiency. Consider the variables that significantly impact the energy demands of a commuter vehicle: highly variable speeds and traffic conditions, unique individual human driving habits, and elevation changes throughout the commute. Each of these dynamics significantly impacts the design of an optimal control system for a combination supercapacitorbattery energy storage system.

The supercapacitor could be sized so that enough energy is available for either an arbitrary acceleration (whether to increase speed or altitude), or deceleration (regenerative braking). Not knowing what the next segment of travel will present would require the employment of a large supercapacitor that is kept essentially "half full." This would be expensive both in weight and money. Therefore, predicting the demands that are about to be placed on the energy storage

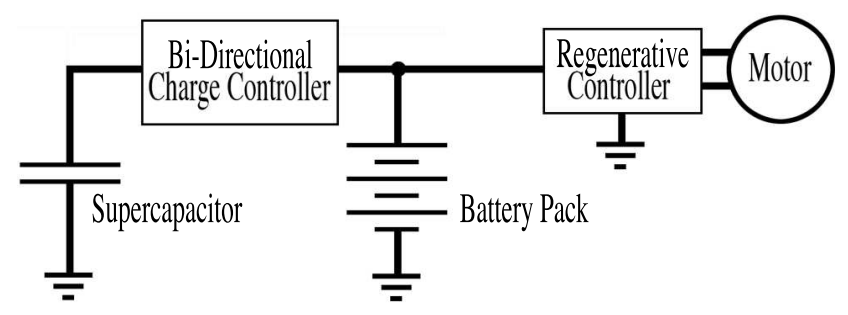

Fig. 1. Heterogeneous energy storage system for electric vehicles system is paramount to minimizing the size and cost of the supercapacitor.

In this novel approach, there are a number of techniques we could employ for predicting the next-segment-of-travel energy demand or recovery that are based on a priori topographical data for the surrounding area, and locations of traffic flow controls (e.g., traffic lights and stop signs). Knowing the slope of the subsequent road segment enables optimization of capacitor energy level on the basis of expected power. For example, when approaching an uphill climb, it is desired to have a full supercapacitor from which to draw the high current this climb demands. On the other hand, when approaching a downhill segment, it is desirable to have an empty supercapacitor in preparation of most efficiently recovering the regenerated energy from traction motor braking. By the same token, a stop sign ahead suggests discharging the supercapacitor before braking in order to maximize regeneration energy recovery. Having this temporal prescience allows spending time-energy budgets wisely to manage charge between the supercapacitor and the battery, especially to limit the battery rate of charge and discharge.

The key enabler for an intelligent energy management system is the ability to create power demand profiles by combining calibrated energy models with personal route history and libraries of actual commute parameters, including topography, trajectory and traffic information. We have developed data collection techniques using vehicle-borne GPS units and maximum-fidelity USGS elevation data for the entire United States, and implemented a world-wide-web repository to collect these data from volunteer commuters (We have received over 9000 miles of real-world commute data to date as described below). Such a repository allows testing of various energy management strategies on a wide sample of typical driving schedules, yielding a more robust solution than training on the FUDS cycle alone.

\section{Energy Management Algorithm Simulation AND TESTING ENVIRONMENT}

A robust electric vehicle model and simulator are required to both utilize commute data from petrol vehicles collected by the project as well as rapidly prototype and test new energy management algorithms. The model translates GPS coordinates from our crowd-sourced dataset into accurate power demands for a replacement electric vehicle, which are then fed into each power management algorithm, along with vehicle, battery, and supercapacitor state. The algorithm decides how to react: how to meet the electric motor power budget and how to control additional current flow between traction battery pack and supercapacitor cache.

\section{A. Real-World Data Collection}

While electric vehicle research programs often rely on Federal driving schedules such as the Urban Driving Schedule as exemplars of vehicle speed over time during typical commutes, this data is insufficiently realistic for training and testing algorithms for active power management. This 
federal data is not a collection of actual commutes but, rather, an abstraction of a typical commute and further lacks all elevation data. Because active energy management must explicitly predict behavior at the specific-commute level, we undertook an extensive data collection program to collect a corpus of real-world commute data to be used explicitly for electric vehicle research. This set aims to yield a more complete sample of typical driving routes, beyond what a single sample could ever hope to cover. Data, collected from hundreds of volunteers internationally, is verified and privatized before ultimately being run through our model and openly published as part of our commuting dataset at www.chargecar.org. The data is generated by GPS devices recording the position of a vehicle each second during each trip. The strength and completeness of the dataset is vital to translating simulated successes into real-world efficiency gains and overall impact.

In addition to the general dataset, we also are building an extensive dataset from a small subset of drivers, who personally agreed to document every trip taken in their vehicle, starting six months ago. This gives data with robust driver history, including behaviors, style, typical routes, and traffic patterns. It is with this subset of data we test our adaptive algorithms that hope to improve efficiency over the life of the vehicle.

\section{B. Robust Electric Vehicle Model Development}

A high-fidelity physics-based model for the electric vehicle enables the transformation of actual commute data from petrol vehicles to simulated electric vehicle commute data for the development of algorithms for our system. It takes, as input, successive timestamped GPS positions and elevations, and it outputs the speed, accelerations, and power demands over time for each trip using a typical longitudinal vehicle model. Using basic principles of physics, we can calculate the force output by the motor from the sum of total forces and resistive forces.

$$
\begin{aligned}
F_{\text {motor }} & =F_{\text {net }}-F_{\text {resistive }} \\
F_{\text {resistive }} & =F_{\text {air }}+F_{\text {roll }}+F_{\text {gravity }}
\end{aligned}
$$

While the net force shown in (1) is simply calculated from the mass of the vehicle and observed acceleration: $F_{n e t}=$ $m a$, the resistive force (2) is composed of air resistance, rolling resistance, and gravitational resistance on slopes.

$$
\begin{aligned}
F_{\text {air }} & =\frac{1}{2} \rho v^{2} C_{d} A \\
F_{\text {roll }} & =C_{r r} m g \\
F_{\text {gravity }} & =m g \sin \theta
\end{aligned}
$$

where,

$\begin{array}{ll}\rho & \text { Air density } \\ v & \text { Vehicle velocity } \\ C_{d} & \text { Vehicle coefficient of drag } \\ A & \text { Vehicle vertical cross section area } \\ C_{r r} & \text { Coefficient of rolling resistance of tires } \\ m & \text { Mass of the vehicle }\end{array}$

$g \quad$ Acceleration due to gravity

$\theta \quad$ Angle of slope

Once the force exerted by the motor is calculated from the net and resistive forces, we can deduce the power required from the power system by the motor,

$$
\text { Power }= \begin{cases}(1 / 0.85) v F_{\text {motor }} & F_{\text {motor }}>0 \\ (0.35) v F_{\text {motor }} & F_{\text {motor }}<=0\end{cases}
$$

In the positive case (6a), where the motor must exert force, we assume an $85 \%$ drivetrain efficiency, with losses due to power transmission, friction, heat loss, etc. The negative case (6b), or during regenerative braking, assumes a $35 \%$ recovery of energy, as witnessed in our initial vehicle tests.

\section{Model Validation}

As previous literature examined on electric vehicles largely used the National Highway Traffic Safety Administration's published driving schedules, which we found to be inadequate, our model was conceived from basic physics principles. It was necessary to validate our model before drawing any significant conclusions from its use. Our first was using a production 2002 Toyota Rav4 EV, and second using Bombardier's large scale people mover vehicles.

1) Toyota Rav4 EV: For our initial validation, we used a GPS device to gather data on a commute while simultaneously scraping power draw data directly from the modified OBCD-II port of a production Toyota Rav4 EV. We passed the GPS data through our model and compared predicted power profiles to the actual measured power output from the vehicle's onboard diagnostics system, shown in Fig. 2. The power values coincide extremely well, with some deviations due to noise on the GPS readings, and some reduced regeneration peaks due to friction braking by the driver. The sections of draw from the motor show almost no deviation. Further model validation with another vehicle would demonstrate the portability and scalability of the system.

2) Bombardier People Transport: Further model validation was approached with the help of Bombardier Inc., who were interested in our intelligent management of the

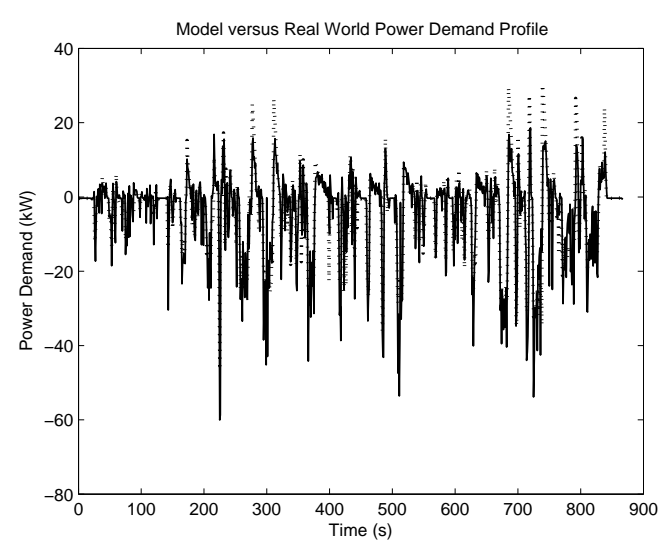

Fig. 2. Model validation using a Toyota Rav4 EV 


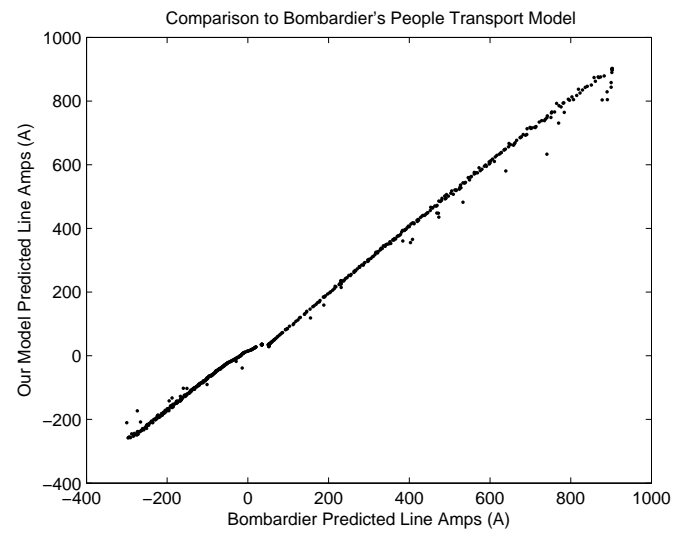

Fig. 3. Model validation from Bombardier's people transport

heterogeneous energy storage system for their large-scale people movers and light rail vehicles. Bombardier provided position data and their power model's output from their extensively developed proprietary train simulator. Their model has undergone extensive validation with real-world systems, and has been depended upon for large-scale engineering decisions for decades. The correlation is shown in Fig. 3, with our model output on the vertical axis and Bombardier's predicted amperes drawn from the power-line on the horizontal axis. An exact correlation between our models would yield points on a perfect diagonal line between the axes. We show a strong correlation with a pronounced diagonal trend in the figure, with only a few deviations yet unresolved. The correlation, coupled with our small vehicle data, suggests that our model is portable between different vehicle types, and scales well for various sizes and weights, the vehicle in question weighing over 28 metric tons.

\section{Intelligent Management Algorithm}

The final piece of the puzzle is the algorithm, or policy, for predicting the upcoming duty and managing the heterogeneous energy storage system. Once the simulator has passed the data through the model to generate a power demand profile over the course of the trip, the management algorithm is used to determine how the motor demand (either draw or regeneration) is met between the battery and supercapacitor. Additionally, it can choose to move charge between the battery and supercapacitor as a degree of freedom to exploit in order to maximize efficiency. The system is only given the starting position and driver identification at the start of the trip, then each data point is input sequentially to mimic real-world data obscurity where the future is not known. At the completion of the trip, the results are compared to a conventional homogeneous traction battery system to measure efficiency gains.

\section{Energy System Algorithm Development}

A heterogeneous energy storage system composed of moderate-rate secondary batteries and supercapacitors leverages the best qualities of each energy storage technology only when properly managed. The challenge is to manage energy across this system for optimal efficiency and battery longevity. Specifically, how is the state of charge of the supercapacitor optimally managed?

The degree to which optimal energy management can decrease overall power duty as seen by the battery has direct impact on both total-cost-of-ownership and initial battery cost. Employing a medium-rate, thick-electrode LiIon battery, or even potentially a low-rate battery such as NiMH or lead-acid, becomes practical when the control algorithms impose appropriate rate demand limits on the battery. This lowers the cost of the single most expensive component of an electric vehicle. Furthermore, a decrease in actual rate spikes seen by the battery as well as total power duty over time should significantly increase thermal stability, additionally increasing the total life of the battery subsystem.

\section{A. Optimal Omniscient Policy}

Our first exploration was toward an optimal control policy, given the entire power demand profile of the trip ahead of time. Using this foreknowledge, the algorithm simply uses a dynamic program to brute forces the power flows at each step between battery, motor, and capacitor to minimize the current-squared integral. The results, shown in Fig. 4 and Table I serve as a bound on possible performance of real-time algorithms, suggest possible successes of our algorithms, and also help to size the supercapacitor for our real-world experimental test vehicle.

We use current-squared demand on the battery as an indicator of efficiency, as it is closely related to both internal efficiency as well as overall battery longevity[5]. The $70.06 \%$ reduction of current-squared suggests extensive battery longevity savings, the measuring of which is beyond the scope or capabilities of our project, as well as significant range increases for most battery chemistries. A simple Peukert model of a battery is used to measure potential range increases, outlined in Equation 7.

$$
I_{\text {effective }}=I_{\text {requested }}^{k}
$$

Where $I_{\text {effective }}$ is the effective current drawn from the battery chemistry, $I_{\text {requested }}$ is the current drawn from the terminals, and $k$ is the Peukert constant. In the case of a leadacid battery, our tests reveal the Peukert constant to be about 1.12. We use a lead-acid battery to emphasize the immediate effect a heterogeneous energy store can have using low-rate batteries.

The $15.54 \%$ range increase is substantial, more than making up for the lost space required by the supercapacitor.

TABLE I

Simulated Algorithm Results

\begin{tabular}{|c||c||c|}
\hline Algorithm & Current Squared Reduction & Range Increase \\
\hline Simple Buffer & $9.05 \%$ & $6.27 \%$ \\
\hline Static Charger & $40.15 \%$ & $9.55 \%$ \\
\hline Contest Leader & $45.50 \%$ & $10.43 \%$ \\
\hline k-Nearest Neighbor & $57.69 \%$ & $12.53 \%$ \\
\hline Optimal Bound & $70.06 \%$ & $15.98 \%$ \\
\hline
\end{tabular}




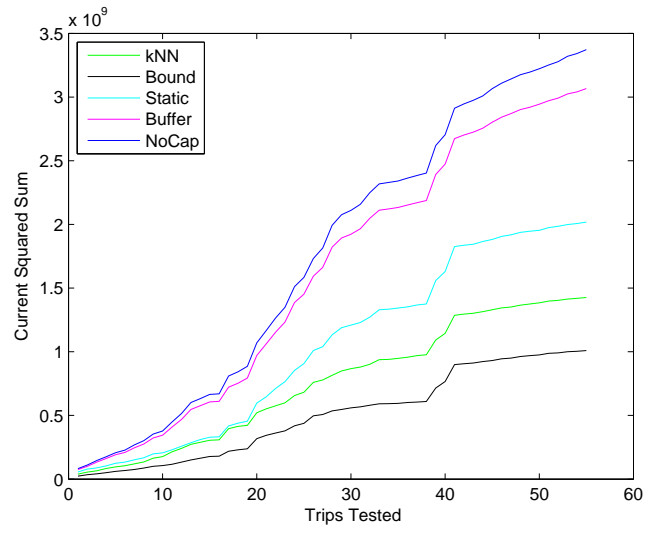

Fig. 4. Comparison of current-squared sum on battery

Such an increase would allow reduced sizes of battery packs to meet the same range requirements, reducing the weight of vehicles to further improve system efficiencies. However, more expensive battery chemistries offer better Peukert coefficients, and such a large range increase would not be realized by this property alone. It is possible, though unexplored, that more significant range increases will be obtained from increased thermal stability.

\section{B. Initial Exploration of a Simple Buffer and Speed Limited Charger}

Another crucial point of reference would be the naive buffer that uses the supercapacitor whenever possible, but never utilizes the potential to move charge between the energy subsystems. That is, whenever there is regeneration, the charge is stored in the supercapacitor, with any overflow being handled by the battery. For acceleration, the supercapacitor is used first until empty, then the battery satisfies the remaining demand. The modest $9.05 \%$ reduction in current-squared on the battery, and the $6.27 \%$ increase in range, suggest significant gains to be had from intelligently exploiting the overlooked degree of freedom. Ultimately, this has limited success due to the inefficiencies of regenerative braking and resistive losses: the energy recovered is a fraction of the energy required to accelerate the vehicle back up to speed.

The first hand-crafted algorithm developed was based off of this simple buffer, but would boost the supercapacitor at a constant rate whenever there was not a large draw on the system, up to a varying maximum charge limit for the supercapacitor. The shifting limit was placed such that, given the current speed of the vehicle, leave exactly enough room in the supercapacitor to store the potential energy regenerated if the vehicle were to come to a complete stop. This was designed to prevent regeneration from overflowing and creating additional duty on the battery subsystem, as well as to keep the capacitor nearly empty at high speeds when acceleration was unlikely and mostly full at low speeds when acceleration was imminent.

Some very intuitive behaviors evolved from this basic set of rules and limited information, such as charging the capacitor to full while stopped at a traffic signal, ready to handle the acceleration immediately to follow. The algorithm was quite successful, over half the reduction of the optimal bound, with a $40.15 \%$ reduction in battery currentsquared, and a $9.55 \%$ increase in battery pack range. The exploitation of the heterogeneous system yielded significant efficiency gains, with no direct knowledge of maps, routes, traffic signals, or driver histories. More advanced hand-built algorithms that incorporate more of these complex features could be extremely successful.

\section{Application of Traditional Machine Learning Techniques}

The best current algorithm utilizes a k-Nearest Neighbor search to predict the upcoming duty on the vehicle. At each time-step in the current trip, the algorithm conducts a search for the $\mathrm{k}$, in our case, 7, nearest, most similar, points in the complete driver's history. Distance, or similarity, is calculated using a weighted combination of features including: GPS coordinates, slope, elevation, speed, acceleration, current power demand, and total duty so-far. GPS coordinates are the most heavily weighted, so stored points in driving history geographically close to the current position are often the chosen neighbors.

In our tests, the most recent 200,000 points, about 55 hours of driving, are used for the history of each driver. Each point stores its true upcoming duty curve that was seen after it was encountered. The nearest-neighbor search finds the most similar neighbors and then averages these upcoming duty curves with each other at each time slice into the future, districtized to one second. This yields an average future duty for seven points in a drivers history most similar to his current state. This is used as the predicted duty for the energy management algorithm, which then simply assumes that this upcoming duty is absolute truth and calculates the same brute force solution as in the optimal bound. Therefore, the gap between the optimal bound and the performance of this algorithm hinges simply on the accuracy of the predictions.

The k-Nearest Neighbor search, which has very little semantic knowledge of the problem, nevertheless yields decent results: a $57.69 \%$ reduction in battery current-squared, and a $12.53 \%$ increase in range. It is successful, moreso on repeated routes, but does not initially succeed in new territory. Over the lifetime of a vehicle, this would continue to improve as more historical data is gathered and better coverage is obtained. Note that this algorithm uses no external data sources or features; additional information and features in the history and current time-step would yield more successful results.

\section{Crowd-sourced Algorithm Search}

The final algorithm we briefly tested was crowd-sourced from a programming contest created for this project. Given the virtually unlimited set of possible features due to internetconnectivity and publicly available data services, we sought out a means to search the possible algorithm space in a massively parallel manner to gather as many perspectives 
and intuitions as possible. The contest rewards work with recognition and prize incentives, but also adopts an opensource nature to encourage collaboration and rapid knowledge integration.

The algorithm is an adaptation of the Speed Limited Charger algorithm with a variable rate of charge. This variable rate of charge is determined from a lookup table calculated from driver histories, using only the current speed and power demand as features. There is still significant room for improvement, with a reduction in current-squared at $45.50 \%$, and could yet yield interesting intuitions or features for the problem.

\section{CONCLUSiOnS}

Our analyses indicate that significant improvements may be realized in the overall cost of ownership of an electric vehicle by addressing energy management at the systems level.

Modeling the ideal case, which defines the limits of efficiency gain, gives a maximum possible reduction of battery losses due to the current duty of $70.06 \%$. This also indicates a maximum range increase of $15.54 \%$. Our analysis using data from real-world commutes and using the best algorithm we have so far shows a practical reduction of battery losses due to the current duty of $57.69 \%$, and a simulated range increase of $12.53 \%$.

Therefore, an heterogeneous architecture controlled with the presented predictive energy management algorithm can significantly reduce battery losses and duty. This allows both smaller battery packs and less-exotic battery chemistries to be used, significantly lowering initial vehicle cost. Additionally, by extending the battery life, this allows the battery cost to be amortized over a significantly greater vehicle longevity.

Such a change at the basic level of cost, through careful systems engineering and predictive modeling, can make electric vehicles significantly more efficient and less costly to produce and operate. It is crucial to make initial cost and total cost-of-ownership practical for the majority of urban commuters. Affordability and practicality are paramount to the growth of this sector, and our work contributes to improving electric vehicles on both fronts.

\section{ACKNOWLEDGMENTS}

The authors gratefully acknowledge the contributions of Bombardier Inc., The Heinz Endowments, Donna Auguste and David Hayes, the ChargeCar team, the CREATE Lab, our international commute data volunteers, and reviewers' comments

\section{REFERENCES}

[1] R. A. Double and S. Liu, "Power and Life Extension of BatteryUltracapacitor Hybrids",IEEE Trans. on Components and Packaging Technologies, vol. 25, no. 1, 2002

[2] H. Zhao and A. F. Burke, "Fuel Cell Powered Vehicles Using Supercapacitors: Device Characteristics, Control Strategies, and Simulation Results", Institute of Transportation Studies, University of California, Davis, Research Report UCD-ITS-RR-10-01, 2010

[3] "Comparing the Benefits and Impacts of Hybrid Electric Vehicle Options for Compact Sedan and Sport Utility Vehicles", EPRI, Palo Alto, CA, Tech. Rep. 1006892, 2002
[4] J. M. Miller et al., "Ultracapacitors as Energy Buffers in a Multiple Zone Electrical Distribution System", Maxwell Technologies, San Diego, CA, White Paper, 2004

[5] S. B. Peterson et al., "Lithium-ion Battery Cell Degradation Resulting from Realistic Vehicle and Vehicle-to-grid Utilization", Journal of Power Sources, vol. 195, 2010, p. 2385-2392 